\title{
Mesure par la méthode des sachets mobiles du taux de disparition de la matière sèche et de l'azote des contenus intestinaux chez des chèvres recevant des pailles traitées chimiquement
}

\author{
F Laurent, J Hasna, B Vignon
}

ENSAIA, INRA, sciences et techniques des productions animales, 2, avenue de la Forêt-de-Haye, 54500 Vandœuvre, France

\begin{abstract}
Summary - Measure of dry matter and nitrogen disappearance of intestinal contents by the mobile bag method in goats fed chemically treated straw. Intestinal disappearance of dry matter and nitrogen from mobile bags of several fractions of duodenal and ileal contents was studied in goats fed chemically-treated straw or ammoniated maize silage. Disappearance of nitrogen in the large intestine was relatively high for treated straw $(50 \%)$ and could explain the low utilization of nitrogen measured with goats.
\end{abstract}

La technique des sachets mobiles a été utilisée chez la chèvre pour étudier le rôle respectif de l'intestin grêle et du gros intestin dans la disparition des fractions azotées de différents fourrages traités chimiquement par de l'ammoniac ou des amines primaires et pour tenter d'expliquer la mauvaise utilisation de l'azote de ces aliments par les ruminants.

Matériel et méthodes - Quatre chèvres munies de canules du rumen et de l'iléon ont successivement reçu à volonté, 2 fois/jour, les 3 fourrages suivants: paille traitée par de l'urée (U) ou de l'éthylènediamine $(E)$, ou ensilage de maïs traité par l'ammoniac $(M)$. Leurs teneurs en azote total (\% de la MS) étaient de $1,25,2,14$ et $2,0 \%$, et celles en azote de la fraction NDF, de $0,49,0,59$ et $0,34 \%$.

Les animaux ont ingéré en moyenne 61 , 31 et $60 \mathrm{~g} \mathrm{MS} / \mathrm{kg} \mathrm{P}^{0,75}$. Pendant 3 jours consécutifs, 4 prélèvements/j (heures décalées entre j) des contenus de l'iléon puis du duodénum ont été réalisés. Après regroupement, chaque échantillon moyen a été lyophilisé et soumis à 2 fractionnements, l'un par traitement dans une solution de détergent neutre (Mason, 1969), qui permet la séparation d'un résidu constitué de parois végétales pauvres en $\mathrm{N}$ soluble et en microbes, et l'autre par filtration et centrifugation (particules alimentaires, Faichney, 1980). Les échantillons duodénaux et iléaux de contenu total, des résidus Mason et des résidus Faichney ont été transférés $(0,2 \mathrm{~g}$ de MS) dans des sachets de nylon (taille $40 \times 15 \mathrm{~mm}$, pores de $50 \mu \mathrm{m}, 4$ répétitions) qui ont été introduits, après passage à l'eau $\left(5 \mathrm{~min}\right.$ à $\left.39^{\circ} \mathrm{C}\right)$, par les canules correspondantes puis récupérés par les fèces. Les quantités de $\mathrm{MS}$ ef d' $\mathrm{N}$ contenues dans les échantillons introduits et récupérés ont été dosées.

La mesure du taux de disparition (TD) (exprimé en \% de la quantité de matière introduite) dans l'intestin total (IT) et dans le gros intestin (GI) permet de calculer le TD dans l'intestin grêle (IG) à l'aide de la relation : TDIG $=100$ (TDIT-TDGI) $/(100-$ TDGI).

Résultats et discussion - Le TD de la MS du contenu de duodénum dans I'IT est plus élevé pour l'ensilage de maïs $(76,3 \%)$ que pour les pailles $U$ et E $(68,4$ et $64,8 \%)$. Pour le contenu d'iléon, le TD de la MS dans le Gl est respectivement égal à $59,5,56,7$ et $51,1 \%$. D'après ces mesures, pour $100 \mathrm{~g}$ de MS duodénale, il disparaîtrait $42 \mathrm{~g}$ dans I'IG et $34 \mathrm{~g}$ dans le $\mathrm{Gl}$ pour le fourrage $M$; ces valeurs sont respectivement de 27 et $41 \mathrm{~g}$ pour la paille $\mathrm{U}$, 
de 28 et $36 \mathrm{~g}$ pour la paille E. La disparition de MS dans I'IG est donc plus faible pour les pailles que pour $M$. Le TD de la MS des résidus Mason et Faichney est dans tous les cas inférieur à $3 \%$.

Les teneurs en $\mathrm{N}$ du contenu duodénal ou iléal et de leurs résidus Mason sont peu différentes entre les régimes (tableau 1). La teneur en $\mathrm{N}$ du résidu Faichney est particulièrement faible pour M. Le TD dans I'IT de I'N du contenu duodénal est très élevé quel que soit le fourrage (tableau I). Pour le contenu iléal, le TD de l'N dans le Gl est égal à 88,85 et $80 \%$ pour $M, U$ et $E$. A partir des TD de la MS et de I'N, le calcul montre que pour $100 \mathrm{~g}$ d' $\mathrm{N}$ de contenu duodénal, les quantités disparues dans I'IG et le GI sont respectivement égales à 67 et $29 \mathrm{~g}, 47$ et $45 \mathrm{~g}, 40$ et $48 \mathrm{~g}$ pour $M$, $U$ et $E$. Le rôle du GI dans la disparition de $\mathrm{N}$ serait donc plus important pour les pailles que pour le maîs.

Le TD de I'N des résidus Mason du contenu duodénal dans l'IT est variable selon les régimes; celui du contenu iléal dans le Gl est très faible avec les pailles, mais relativement élevé pour $M$ (tablıau 1). D'après ces résultats, I'IG aurait une part prépondérante par rapport au GI dans la disparition de l' $N$ des résidus Mason duodénaux pour U (48 contre $4 \%$ ) et pour E (19 contre $10 \%$ ) mais la tendance est inversée pour $M$ (15 contre $33 \%$ ). Les valeurs particulièrement faibles obtenues avec la paille $E$ s'expliquent par la réduction très marquée de la fraction insoluble dans le détergent neutre après traitement (Laurent et al, 1985). Les TD de I'N des résidus Faichney sont supérieurs à ceux des résidus Mason mais la hiérarchie entre les segments de l'intestin est identique pour $U$ et $E$ (tableau 1).

L'utilisation de cette technique a permis de hiérarchiser entre IG et GI le TD de I'N total et de l'azote des résidus Mason et Faichney. La faible valorisation chez la chèvre de l'azote des pailles traitées aux amines (Laurent et al, 1985) est sans doute liée au rôle relatif du Gl qui représente respectivement près de 49 et $55 \%$ de la disparition totale pour les pailles $U$ et $E$. Le fait que le TD de la fraction azotée liée au résidu Mason, qui représente $25 \%$ de l'azote total, soit également faible, contribue à expliquer cette mauvaise utilisation de l'azote.

Faichney GJ (1980) Austr J Agric Res 31, 1129-1137

Laurent F, Blanchard G, Vignon B (1985)

Anim Feed Sci Technol 13, 131-140

Mason VC (1969) J Agric Sci Camb 73, 99111

Tableau I. Teneurs en azote des contenus duodénaux et iléaux, et taux de disparition de l'azote des contenus duodénaux dans l'intestin total, et de l'azote des contenus iléaux dans le gros intestin.

\begin{tabular}{|c|c|c|c|c|c|c|c|c|c|c|c|c|}
\hline & \multicolumn{6}{|c|}{ Contenu de duodénum } & \multicolumn{6}{|c|}{ Contenu d'iléon } \\
\hline & \multicolumn{2}{|c|}{$\begin{array}{c}\text { Contenu } \\
\text { total }\end{array}$} & \multicolumn{2}{|c|}{$\begin{array}{l}\text { Résidu } \\
\text { Mason }\end{array}$} & \multicolumn{2}{|c|}{$\begin{array}{c}\text { Résidu } \\
\text { Faichney }\end{array}$} & \multicolumn{2}{|c|}{$\begin{array}{c}\text { Contenu } \\
\text { total }\end{array}$} & \multicolumn{2}{|c|}{$\begin{array}{l}\text { Résidu } \\
\text { Mason }\end{array}$} & \multicolumn{2}{|c|}{$\begin{array}{l}\text { Résidu } \\
\text { Faichney }\end{array}$} \\
\hline & 1 & 2 & 1 & 2 & 1 & 2 & 1 & 2 & 1 & 2 & 1 & 2 \\
\hline $\begin{array}{l}\text { Paille } \mathrm{U} \\
\text { Paille } \mathrm{E} \\
\text { Mails } \mathrm{NH}_{3}\end{array}$ & $\begin{array}{l}2,65 \\
2,61 \\
3,13\end{array}$ & $\begin{array}{l}92 \\
88 \\
97\end{array}$ & $\begin{array}{l}0,83 \\
0,63 \\
0,72\end{array}$ & $\begin{array}{l}52 \\
29 \\
48\end{array}$ & $\begin{array}{l}1,1 \\
1,1 \\
0,1\end{array}$ & $\begin{array}{l}59 \\
44 \\
71\end{array}$ & $\begin{array}{l}1,53 \\
1,75 \\
1,80\end{array}$ & $\begin{array}{l}85 \\
80 \\
88\end{array}$ & $\begin{array}{l}0,52 \\
0,58 \\
0,60\end{array}$ & $\begin{array}{r}7 \\
12 \\
39\end{array}$ & $\begin{array}{l}0,49 \\
0,72 \\
-\end{array}$ & $\begin{array}{l}13 \\
19 \\
-\end{array}$ \\
\hline
\end{tabular}

1 : Teneur en azote en $\%$ de la MS; 2 : taux de disparition de l'azote (\%). 